Revista Digital Universitaria

Vol. 23, Núm. 1, enero-febrero 2022

\title{
Filosofía para niños y adolescentes desde la Neuroeducación
}

\author{
Axel Rubalcava Gámez y Pablo Luis Hernández Adame
}

\section{Resumen}

En este artículo, primeramente, se explica de manera concisa la dualidad sociedad-educación, que conlleva que los cambios sociales impactan gradualmente en los educativos y viceversa, dichos cambios dan origen a proyectos tanto sociales como educativos, de estos últimos se describen dos que han sido revolucionarios en las últimas décadas, por un lado, la Filosofía para niños y adolescentes de Matthew Lipman y Ann Margaret Sharp, y por el otro, la Neuroeducación. Después, se comprende el primer proyecto educativo mencionado desde los hallazgos científicos del segundo. Finalmente, se plantea la cuestión de que, si se puede hablar del surgimiento de un nuevo paradigma que podría denominarse Neuroeducación Filosófica o Neurofilosofía para niños y adolescentes o simplemente hablar de ambos proyectos educativos por separado, pero sin dejar de lado la comprensión hecha de uno sobre la mirada del otro.

Palabras clave: Filosofía para Niños y Adolescentes, pensamiento multidimensional, comunidad de diálogo filosófico y Neuroeducación.

\section{NEUROPHILOSOPHY FOR CHILDREN AND ADOLESCENTS: STIMULATING MULTIDIMEN-}

\section{SIONAL THINKING}

\begin{abstract}
In this article, first, the society-education duality is concisely explained, which implies that social changes gradually impact educational ones and vice versa, these changes give rise to both social and educational projects, of the latter, two projects are described that are being revolutionaries in the last decades, on the one hand, Philosophy for Children and Adolescents of Matthew Lipman and Ann Margaret Sharp, and on the other, Neuroeducation. Afterwards, the first educational project mentioned is understood from the scientific findings of the second. Finally, the question arises as to whether it is possible to speak of the emergence of a new paradigm that could be called Philosophical Neuroeducation or Neurophilosophy for Children and Adolescents or simply to speak of both educational projects separately, but without neglecting the understanding made of one on the gaze of the other.
\end{abstract}

Keywords: Philosophy for Children and Adolescents, multidimensional thinking, community of physiological dialogue and Neuroeducation. 
"Filosofía para niños y adolescentes desde la Neuroeducación" Axel Rubalcava Gámez y Pablo Luis Hernández Adame Vol. 23, Núm. 1, enero-febrero 2022 Revista Digital Universitaria

Axel Rubalcava Gámez oda12340@gmail.com

Es licenciado en Educación Primaria, maestro en Educación y doctorante en Educación, está adscrito en la Seduzac (Secretaría de Educación del Estado de Zacatecas), ha realizado dos veces el diplomado de Filosofía para Niños y Adolescentes, el primero con el CECAPFI (Centro Educativo para la Creación Autónoma en Prácticas Filosóficas) y el segundo con el centro Asertum, ha hecho varios cursos-talleres también de Filosofía para Niños y Adolescentes: curso-taller "Filosofía para niños y adolescentes (Desarrollo del Pensamiento y Formación Valoral)" del ceLAfIN (Centro Latinoamericano de Filosofía para Niños y Adolescentes), curso-taller "Educación socioemocional desde FpN" y curso-taller "El uso de manuales en FpN" del G-Fan (Grupo Filosófico de Adolescentes y Niños), taller "Hannah Mirada y empoderamiento desde las infancias" y taller "Educación con perspectiva de género. Herramientas para la deconstrucción y la inclusión del aula" de Noema Consultores y actualmente está realizando el diplomado Online "Filosofía con enfoque en comunidades de indagación" también con Noema Consultores.

También tiene un canal de YouTube titulado "Filosofía para Niñas, Niños y Adolescentes" donde da a conocer la propuesta pedagógica de Lipman y Sharp.

\section{Pablo Luis Hernández Adame}

pabloyae_2@hotmail.com orcid.org/0000-0003-3554-5898

Fue coordinador del área de posgrado e investigación en la Universidad Autónoma de Fresnillo (UAF). Es maestro en educación por la Benemérita Escuela Normal "Manuel Ávila Camacho". Maestro en Ciencias Nucleares por la Universidad Autónoma de Zacatecas y Doctor en Ingeniería y Ciencia de los Materiales por el Instituto de Física de la Universidad Autónoma de San Luis Potosí. Actualmente, es Posdoctorante en el Instituto de Ciencias Físicas de la Universidad Nacional Autónoma de México, campus Cuernavaca Morelos. Sus trabajos de investigación en el área de la educación giran en torno a la definición de paradigmas de la Universidad Autónoma de Fresnillo y el diseño de clases en línea para la apropiación del modelo educativo de la universidad. En el área de las ciencias exactas sus líneas de investigación son en el estudio y caracterización biofísica de membranas modelo, péptidos antimicrobianos y antivirales para el diseño de fármacos de nueva generación. 
"Filosofía para niños y adolescentes desde la Neuroeducación" Axel Rubalcava Gámez y Pablo Luis Hernández Adame Vol. 23, Núm. 1, enero-febrero 2022 Revista Digital Universitaria

\section{Introducción}

L

a raza humana está en constante evolución, la globalización y la sociedad del conocimiento a través de las ciencias y las tecnologías crecen a pasos agigantados, por lo que requieren individuos capaces de pensar globalmente y al mismo tiempo, que actúen localmente tomando en cuenta las características socioculturales, geográficas, históricas y económicas de la sociedad donde estén inmersos. Es así como, los cambios generales y particulares de la humanidad demandan pedagogías adaptadas y en constante evolución perfectible, es decir, que estén continuamente mejorando, puesto que existe una dualidad sociedad-educación, donde los cambios sociales impactan gradualmente en la educación y viceversa.

En las últimas décadas, más precisamente las de la segunda mitad del siglo $x x$ y las que han transcurrido del siglo xxl, han emergido varios proyectos educativos revolucionarios como la Filosofía para niños y adolescentes y la Neuroeducación, en contraposición de la educación bancaria donde el docente y/o madre/padre de familia como autoridad trasmiten los conocimientos al alumno, éste último desde un rol pasivo, receptivo y no reflexivo (mecánico). No obstante, no ha habido muchos estudios en los que se comprenda el proceso teórico y práctico del primer proyecto educativo mencionado desde los hallazgos científicos del segundo. Por tanto, este artículo pretende, de una manera breve, comprender la Filosofía para niños y adolescentes desde la Neuroeducación.

\section{Desarrollo}

Primeramente, la Filosofía para niños y adolescentes — que tiene su raíz filosófica en el método socrático, el pragmatismo y el pluralismo - es una propuesta pedagógica que se empezó a crear a finales de los años sesenta en Estados Unidos por Matthew Lipman y Ann Margaret Sharp con los objetivos de que los estudiantes - desde el nivel inicial o preescolar hasta el bachillerato-construyan y clarifiquen conceptos filosóficos, desarrollen y practiquen habilidades de pensamiento y exploren y consoliden valores a través de la metodología de la comunidad de diálogo filosófico donde se establecen y evalúan acuerdos de participación democrática, se leen episodios y capítulos de las novelas filosóficas - o se usan otros materiales curriculares—, se lleva a cabo un diálogo filosófico donde se analiza y reflexiona la lectura por medio de preguntas y se enriquece con ejercicios, planes de discusión y actividades contenidas en los manuales para acompañar al facilitador en un ambiente de aprendizaje donde los integrantes se sientan seguros física, socioemocional y mentalmente (Echeverría, 2011).

Por otro lado, la Neuroeducación "es una nueva visión de la instrucción y la educación basada en los conocimientos acerca de cómo funciona el cerebro" (Mora, 2019, p. 29). 
Figura 1. La Neuroeducación (Adaptación de Guillén, 2017)

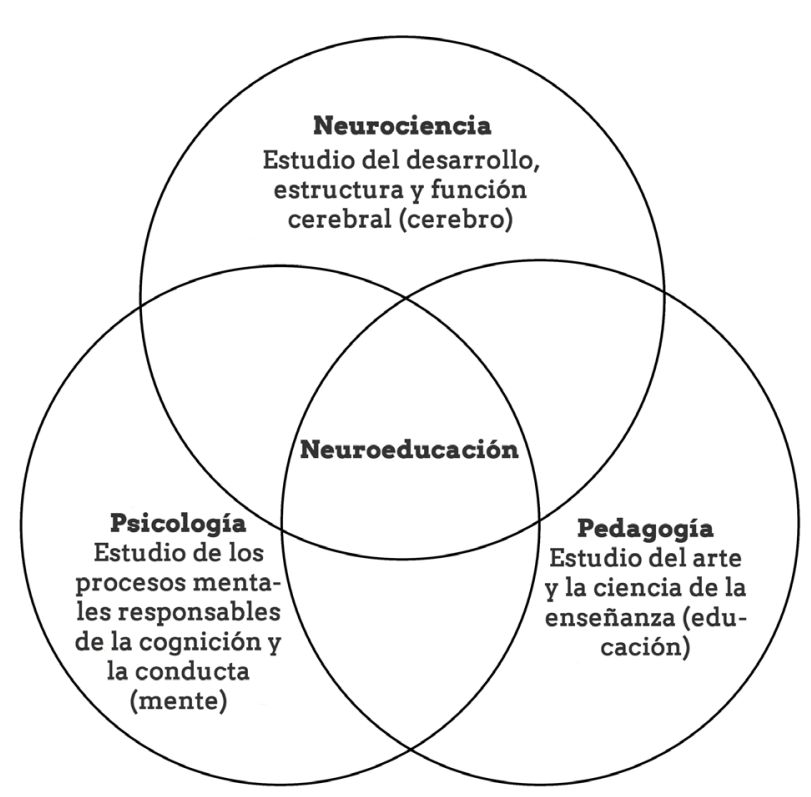

Además, de acuerdo con Francisco Mora (2019), la Neuroeducación apunta a conocer las herramientas que la Neurociencia provee a la educación para hacerla más eficiente: que sirvan para identificar problemas neurológicos y/o psicológicos que impidan o interfieran en el aprendizaje de los estudiantes; que sirvan para formar mejores ciudadanos críticos, creativos y emocionalmente inteligentes; y que ayuden al maestro en mejorar su transposición didáctica, entendida como el proceso en donde se modifica o adapta el saber para hacerlo asequible de ser enseñado.

La forma de pensar, sentir y ser de una persona es el producto del funcionamiento de su cerebro en interacción con el resto de sus órganos y en relación con el medio que lo rodea. Tanto el cerebro como las neuronas que yacen en él son plásticas, es decir, cambiantes; a esta característica se le conoce como neuroplasticidad. Estos cambios neuronales son causados por estímulos que el cerebro, a través de la percepción y los sentidos, capta del medio ambiente. Las características de cada contexto — familiar, educativo y sociocultural- permean en el desarrollo de las áreas cerebrales de cada individuo. Por tanto, cada cerebro humano es diferente debido a su neuroplasticidad (Mora, 2019).

En tanto, las células nerviosas son las que viven en el sistema nervioso donde podemos encontrar neuronas y glías. Las neuronas, por medio de interacciones bioquímicas y eléctricas, producen conexiones llamadas sinapsis, a la formación de estas se le conoce como sinaptogénesis. Las sinapsis tienen la función de comunicar información - que se recibe del medio ambiente- de una neurona a otra, dicha información es transportada por sustancias químicas conocidas como neurotransmisores entre las cuales están: la serotonina, la dopamina, la endorfina, la epinefrina (adrenalina), norepinefrina (noradrenalina), entre otros. Las dendritas son aquellas partes de la neurona, en forma de raíces, 
Video. Qué son las sinapsis. (Cerebrotes, 2019) que se encargan de transmitir y recibir las señales que se producen por las sinapsis. Por otro lado, las glías tienen la función de sostener y nutrir a las neuronas (Mora, 2019). Para mejor entendimiento en la referencia (García, 2019) se presenta un video donde se explica visualmente el proceso de formación de sinapsis o sinaptogénesis.

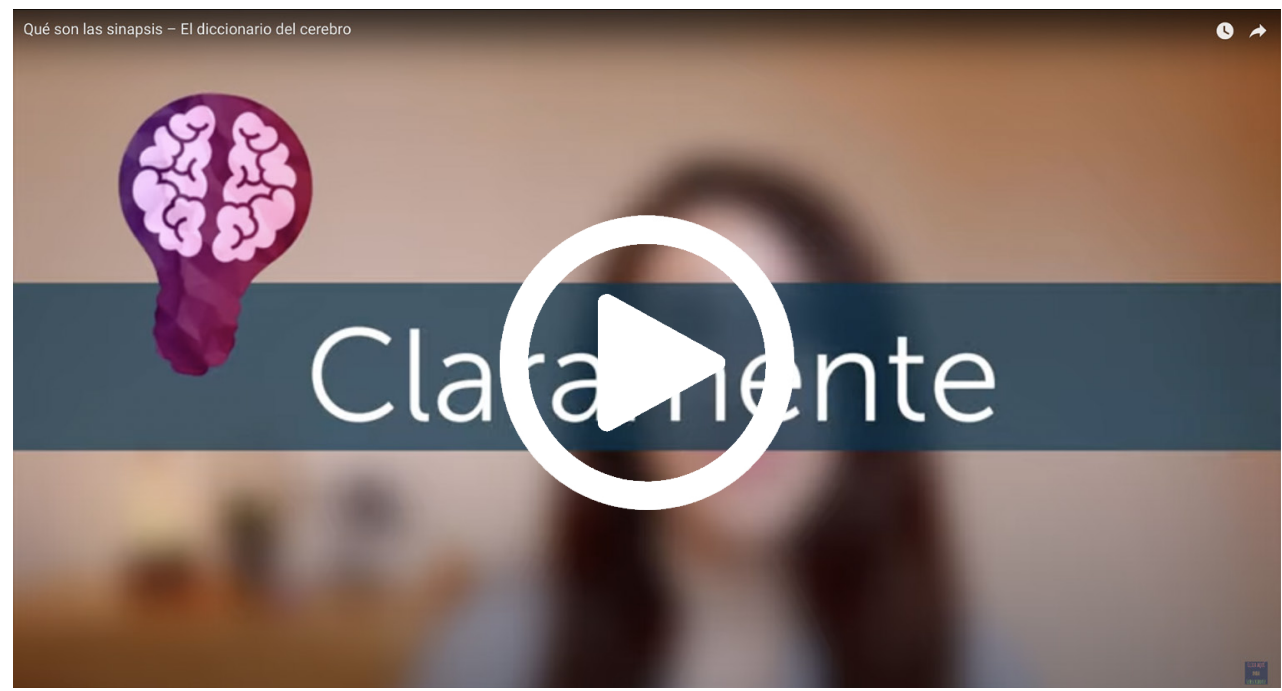

Las funciones de cada neurotransmisor son las siguientes: la serotonina produce felicidad y regula el ciclo sueño-vigilia; la dopamina causa sensaciones placenteras, la regulación de la memoria y el aprendizaje; la endorfina ocasiona una sensación de placer y euforia, promueve la calma y mejora el humor; la adrenalina o epinefrina se encarga de poner alerta al cuerpo; la noradrenalina - norepinefrina está implicada en la motivación; el glutamato es importante para la memoria y la mediación de la información motora, sensorial, cognitiva y emocional; y la acetilcolina participa en los procesos de memoria, asociación y pase de sueño y vigilia (García-Allen, 2016).

El cerebro, de manera general, se divide en tres partes: primitivo, límbico y neocórtex: el cerebro primitivo —o reptil - se encarga de los instintos básicos de supervivencia: deseo sexual, búsqueda de alimentos, el miedo a la muerte, etcétera; el cerebro límbico se ocupa de la afectividad —-sentimientos, emociones y deseos-; y el cerebro neocórtex se responsabiliza de pensar de forma racional, creativa, abstracta e imaginativa (Hinojosa, 2012).

Si el medio ambiente que rodea al individuo es fundamental en el desarrollo de habilidades físicas, cognitivas y socioafectivas producto de la sinaptogénesis — formación de sinapsis o conexiones entre neuronas - y la neuroplasticidad — característica neuronal de estar en constante cambio-, entonces la puesta en práctica de la comunidad de diálogo filosófico donde se usan novelas filosóficas y/u otros materiales curriculares de forma gradual y eficiente produce cambios neuronales en el cerebro del estudiante. Se infiere que la construcción y clarificación de conceptos filosóficos, el desarrollo y practicidad de habilidades de 
"Filosofía para niños y adolescentes desde la Neuroeducación" Axel Rubalcava Gámez y Pablo Luis Hernández Adame Vol. 23, Núm. 1, enero-febrero 2022

Revista Digital Universitaria

pensamiento y la exploración y consolidación de valores son las manifestaciones de la formación de sinapsis y conexiones neuronales estimuladas mediante el diálogo filosófico.

Para lograr lo anterior, el docente debe ser, como dice Lipman, un facilitador "filosóficamente humilde y pedagógicamente fuerte" en donde planifique, implemente y evalúe estrategias pedagógicas y actividades didácticas (estímulos) que atiendan a las necesidades de aprendizaje de los estudiantes manifestadas en su dimensión filosófica para que reaccionen produciendo los neurotransmisores que ayuden a encender la chispa de la curiosidad y la admiración en ellos y así darle paso a que el pensamiento multidimensional se estimule.

De acuerdo con Echeverría (2011), el pensamiento multidimensional es la meta educativa de la propuesta pedagógica de Filosofía para Niños y Adolescentes, y está formada por tres dimensiones: la primera es la crítica, la capacidad humana para pensar por sí misma; la segunda es la creativa, la habilidad de las personas para inventar cosas, ideas y soluciones ante los problemas; la tercera es la cuidadosa/valorativa, la destreza de la gente para tomar en cuenta a los demás desde los valores, la democracia y la congruencia entre el pensar, sentir, decir y hacer; sin embargo, otra investigación realizada por Leslie Cázares (2014) proponen una cuarta, la colaborativa, que es la capacidad de los individuos para mirar hacia el bien común tomando en cuenta la diversidad de ideas, intereses, necesidades, etc.

Nadie nace filosofando, es decir, nadie viene a este mundo pensando multidimensionalmente - pensando crítica, creativa, cuidadosa/valorativa y colaborativamente-. No obstante, según la neuroeducación, se nace con la potencialidad de filosofar, que sólo con el aprendizaje, producto de la implementación y evaluación de propuestas pedagógicas como Filosofía para niños y adolescentes con su metodología comunidad de diálogo filosófico logra convertir en hecho aquello que estaba únicamente en potencia. Entre más pronto se estimule el pensamiento multidimensional en los estudiantes más provechosos van a ser, puesto que las estimulaciones sinápticas, como afirma Francisco Mora (2019), son más frecuentes de pequeños que de grandes. Por eso Lipman (Echeverría, 2011) mencionó que el pensamiento filosófico se debe empezar a practicar desde pequeño y no esperarse hasta la universidad.

El pensamiento multidimensional se manifiesta a través de las habilidades de pensamiento las cuales se dividen en razonamiento, indagación, formación de conceptos y traducción/transferencia; la dimensión crítica se exterioriza en las habilidades de razonamiento e indagación, la dimensión creativa en las habilidades de indagación y formación de conceptos, las dimensiones cuidadosa/ valorativa y colaborativa en las habilidades de traducción/transferencia (Echeverría, 2011). 
"Filosofía para niños y adolescentes desde la Neuroeducación" Axel Rubalcava Gámez y Pablo Luis Hernández Adame

Vol. 23, Núm. 1, enero-febrero 2022

Revista Digital Universitaria

Tabla 1. Tipos de habilidades de pensamiento (Adaptación de Echeverría, 2011).

Figura 2. Los lóbulos cerebrales (Adaptación de Guillén, 2017).

\begin{tabular}{|c|c|c|c|}
\hline Razonamiento & Indagación & $\begin{array}{c}\text { Formación de } \\
\text { conceptos }\end{array}$ & $\begin{array}{l}\text { Traducción/ } \\
\text { transferencia }\end{array}$ \\
\hline Conversión & $\begin{array}{l}\text { Formación de } \\
\text { hipótesis }\end{array}$ & $\begin{array}{l}\text { Hacer } \\
\text { distinciones }\end{array}$ & Saber escuchar \\
\hline $\begin{array}{l}\text { Hacer Inferencias } \\
\text { adecuadas }\end{array}$ & $\begin{array}{l}\text { Proponer } \\
\text { ejemplos y } \\
\text { contraejemplos }\end{array}$ & $\begin{array}{l}\text { Establecer } \\
\text { conexiones }\end{array}$ & $\begin{array}{l}\text { Ser sensible a } \\
\text { los sentimientos } \\
\text { de los demás }\end{array}$ \\
\hline $\begin{array}{l}\text { Dar buenas } \\
\text { razones }\end{array}$ & $\begin{array}{l}\text { Cuestionar } \\
\text { adecuadamente }\end{array}$ & Definir & $\begin{array}{l}\text { Inferir visiones } \\
\text { del mundo }\end{array}$ \\
\hline $\begin{array}{l}\text { Realizar buenas } \\
\text { analogías }\end{array}$ & Contrastar & $\begin{array}{l}\text { Detectar } \\
\text { similitudes }\end{array}$ & $\begin{array}{l}\text { Tener } \\
\text { imaginación } \\
\text { moral }\end{array}$ \\
\hline $\begin{array}{l}\text { Detectar } \\
\text { presuposiciones }\end{array}$ & Pedir evidencias & Ser razonables & $\begin{array}{l}\text { Tener mente } \\
\text { abierta }\end{array}$ \\
\hline $\begin{array}{l}\text { Pensar } \\
\text { hipotéticamente }\end{array}$ & $\begin{array}{l}\text { Demandar } \\
\text { criterios }\end{array}$ & $\begin{array}{l}\text { Relacionar, } \\
\text { construir con } \\
\text { la ayuda de los } \\
\text { demás }\end{array}$ & $\begin{array}{l}\text { Respetar a los } \\
\text { demás }\end{array}$ \\
\hline $\begin{array}{l}\text { Pensar } \\
\text { silogísticamente }\end{array}$ & $\begin{array}{l}\text { Ser sensible al } \\
\text { contexto }\end{array}$ & & $\begin{array}{l}\text { Poner el ego en } \\
\text { perspectiva }\end{array}$ \\
\hline $\begin{array}{l}\text { Detectar } \\
\text { contradicciones }\end{array}$ & Autocorregirse & & $\begin{array}{l}\text { Seguir la } \\
\text { indagación } \\
\text { hacia donde } \\
\text { ésta conduzca }\end{array}$ \\
\hline Estandarizar & $\begin{array}{l}\text { Crear } \\
\text { alternativas }\end{array}$ & & \\
\hline
\end{tabular}

Es evidente que, la naturaleza crítica y creativa de las habilidades de razonamiento, indagación y formación de conceptos se comprenden a través de la neurociencia cognitiva ya que esta investiga el desarrollo, la estructura y el funcionamiento de las áreas cerebrales que se estimulan cuando se ponen en práctica la creatividad y racionalidad.

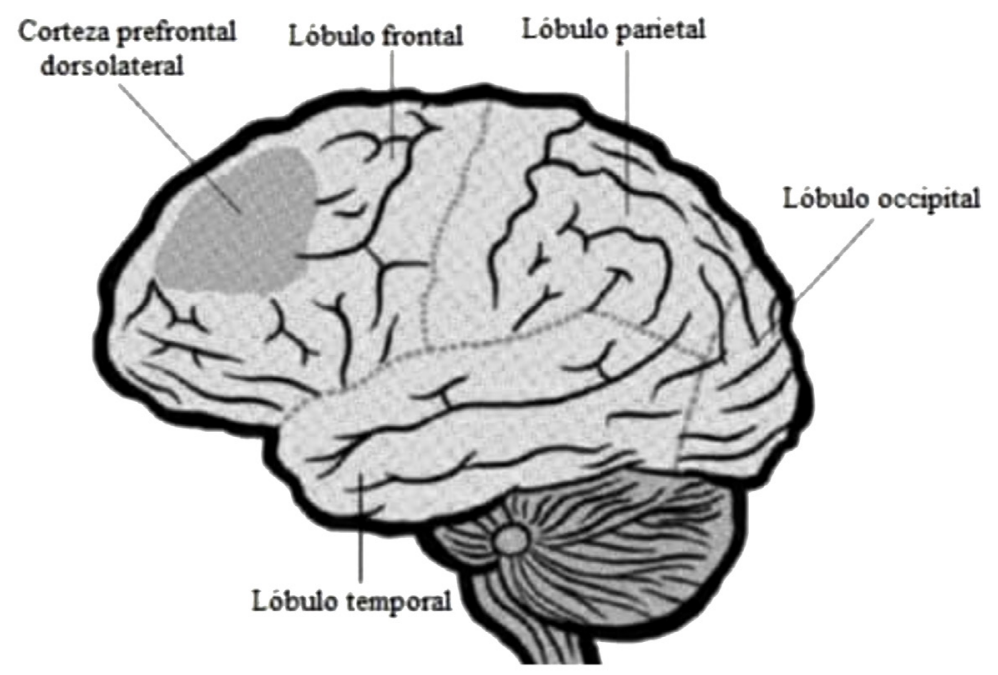


"Filosofía para niños y adolescentes desde la Neuroeducación" Axel Rubalcava Gámez y Pablo Luis Hernández Adame Vol. 23, Núm. 1, enero-febrero 2022

Revista Digital Universitaria

Figura 3. Partes del sistema límbico (Adaptada de Mora, 2017).
Por otro lado, la naturaleza cuidadosa/valorativa y colaborativa de las habilidades de traducción/transferencia se comprenden por medio de la neurociencia afectiva porque ésta estudia las estructuras cerebrales que están implicadas en los procesos emocionales y sentimentales (Goleman, 2016). Figura 3. Se muestran las partes del sistema límbico, que es aquella parte del cerebro que se encarga de la afectividad.

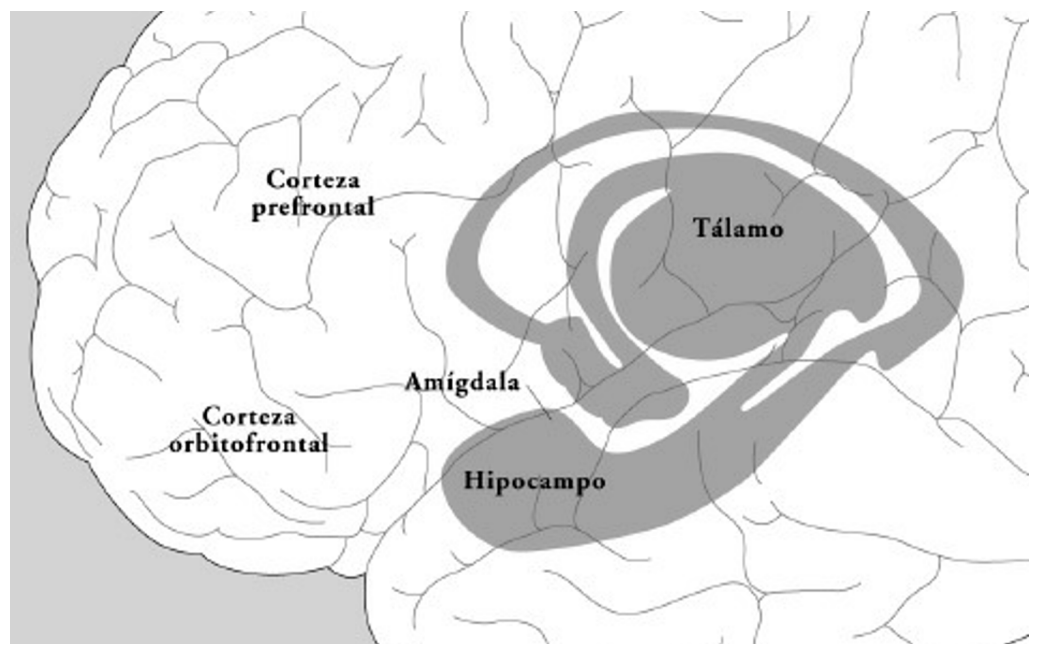

En cuestión de los valores dentro de las neurociencias, nace la Neuroética, que estudia, de forma general, el funcionamiento del cerebro y, en forma particular, los sistemas neuronales que, en interacción con un contexto sociocultural determinado, dan como resultado un ser ético y moral caracterizado por la puesta en práctica de valores tales como la puntualidad, la responsabilidad, la individualidad, el autocontrol, el respeto, la nobleza, la libertad, entre otros (Mora, 2019).

La Filosofía para niños y adolescentes y la metodología de la comunidad de diálogo filosófico tienen la intención de desarrollar y poner en práctica habilidades de pensamiento y explorar y consolidar valores en los estudiantes de educación básica y media superior, por medio de la construcción y clarificación de ideas o conceptos filosóficos y de la práctica consciente de los acuerdos de participación democrática para así, generar hábitos educativos que tienen su origen, como señala Goleman (2016) en los circuitos y redes neuronales que nacen de la neurogénesis, denominada como la capacidad neuronal de producir nuevas células nerviosas como las neuronas; una parte - células madre- se centra en crear más células nerviosas y la otra parte se van a la región del cerebro donde haga falta, transformándose en las células requeridas donde su cometido tiene que ver con el aprendizaje.

\section{Conclusiones}

Es así como se comprende brevemente la Filosofía para niños y adolescentes desde la Neuroeducación, aunque aún falta mucho camino por investigar. La cuestión queda en que, si se puede hablar, a partir de dicha comprensión, de 
"Filosofía para niños y adolescentes desde la Neuroeducación" Axel Rubalcava Gámez y Pablo Luis Hernández Adame Vol. 23, Núm. 1, enero-febrero 2022 Revista Digital Universitaria

una Neuroeducación Filosófica o de una Neurofilosofía para niños y adolescentes o simplemente hablar de ambos proyectos educativos por separado, pero sin dejar de lado la comprensión hecha de uno desde la mirada del otro; dado que el pensamiento multidimensional que plantea Lipman y Sharp se ve sustentado sólidamente por los hallazgos científicos de las neurociencias.

\section{Referencias}

* Cázares, L. (2014). Imbricar Filosofía para Niños y Jóvenes en los centros escolares. Barcelona: Octaedro.

* Cerebrotes. (2019, 19 de diciembre). Qué son las sinapsis - El diccionario del cerebro. [video]. YouTube. https://www.youtube.com/watch?V=DEzT_JM6WfY

* Echeverría, E. (2011). Filosofía para Niños. México D.F.: Ediciones SM.

* García-Allen, J. (2016). Tipos de neurotransmisores: funciones y clasificación. Psicología y Mente. https://cutt.ly/PUw3u1h

* Goleman, D. (2016). El cerebro y la inteligencia emocional: Nuevos descubrimientos. Barcelona: Ediciones B, S. A. .

* Guillén, J. C. (2017). Neuroeducación en el aula. De la teoría a la práctica. USA: Createspace Independent Publishing Platform.

* Hinojosa, M. (2012). Tips para preparar la clase diaria. Conceptos claves para las competencias docentes. D.F.: Trillas.

- Mora, F. (2017). Cómo funciona el cerebro. Madrid: Alianza Editorial.

* Mora, F. (2019). Neuroeducación. Sólo se puede aprender aquello que se ama. Madrid: Alianza Editorial.

\section{Cómo citar este artículo}

* Rubalcava Gámez, Axel y Hernández Adame, Pablo Luis. (2022, enero-febrero). Filosofía para niños y adolescentes desde la Neuroeducación. Revista Digital Universitaria (RDU), 23(1). http://doi.org/10.22201/cuaieed.16076079e.2022.23.1.6 